\title{
Control of Morphology and Subsequent Toxicity of $A \beta$ Amyloid Fibrils through the Dequalinium-induced Seed Modification
}

\author{
Jina Kim, Eun-Kyung Myung, In-Hwan Lee, and Seung R. Paik \\ School of Chemical and Biological Engineering, College of Engineering, Seoul National University, Seonl 151-744, Korea \\ "E-mail: srpaik@smuackr. \\ Received Angust 28, 2007
}

\begin{abstract}
$\Lambda$ myloid fibril formation of amyloid $\beta i \Lambda 4$ protein $(\Lambda \beta)$ is critical to understand the pathological mechanism of $\Lambda$ lzheimer's disease and develop controlling strategy toward the neurodegenerative disease. For this purpose, dequalinium (DQ) has been employed as a specific modifier for $\Lambda \beta$ aggregation and its subsequent cytotoxicity. In the presence of $\mathrm{DQ}$, the final thioflavin-T binding fluorescence of $\Lambda \beta$ aggregates decreased significantly. It was the altered morphology of $\Lambda \beta$ aggregates in a form of the bundles of the fibrils, distinctive from normal single-stranded amyloid fibrils, and the resulting reduced $\beta$-sheet content that were responsible for the decreased fluorescence. The morphological transition of $\Lambda \beta$ aggregates assessed with atomic force microscope indicated that the bundle structure observed with DQ appeared to be resulted from the initial multimeric seed structure rather than lateral association of preformed single-stranded fibrils. Investigation of the seeding effect of the DQ-induced $\Lambda \beta$ aggregates clearly demonstrated that the seed structure has determined the final morphology of $\Lambda \beta$ aggregates as well as the aggregative kinetics by shortening the lag phase. In addition, the cytotoxicity was also varied depending on the final morphology of the aggregates. Taken together, DQ has been considered to be a useful chemical probe to control the cytotoxicity of the amyloid fibrils by influencing the seed structures which turned out to be central to develop therapeutic strategy by inducing the amyloid fibrils in different shapes with varied toxicities.
\end{abstract}

Key Words : A $\beta$, Dequalinium, Seeding effect, Amyloid fïbrils, Alzheimer's disease

\section{Introduction}

Alzheimer's disease $(A D)$ is a representative neurodegenerative disorder predominantly affecting cholinergic neurons with clinical manifestations of cognitive deficits involving memory, orientation, judgment, and reasoning. ${ }^{1-3}$ The brain of $\mathrm{AD}$ patients contains a large number of amyloid deposits known as senile plaques in which amyloid $\beta / A 4$ proteins (A $\beta$ ) with 40 amino acid residues ( $A \beta 40$ ) has constituted the major component along with another extended form of $\mathrm{A} \beta$ with 42 amino acid residues (A $\beta 42){ }^{+-6}$ As suggested by the amyloid cascade hypothesis, ${ }^{7}$ the $A \beta$ aggregates in a fibrillar state have been recognized as a critical phenomenon to understand the molecular etiology of $\mathrm{AD}$. However, the amyloidosis mechanism has not been fully understood; therefore no effective treatment strategy for $\mathrm{AD}$ has been suggested. Many attempts have been made to understand the mechanism and find chemical compounds that influence the amyloid fibril formation reaction of $\mathrm{A} \beta^{8-12}$ Those compounds have been also expected to be used as probes to assess basic assembly mechanism of the peptide leading to the amyloid fibril formation.

Dequalinium (DQ), 1,1'-(1,10-decanediyl)-bis-[4-amino2-methylquinolium] (Fig. 1A), was demonstrated to cause the protofibril formation of $\alpha$-synuclein, a pathological component of Parkinson's disease, by inducing the selective protein self-oligomerization. ${ }^{13}$ The resulting protofibrils were shown to affect cell viability by influencing membrane stability. The compound has been also shown to exert anti- tumor effect by selectively accumulating in mitochondria. ${ }^{14}$ In fact, DQ affects crucial enzymes for cellular biogenesis such as mitochondrial $\mathrm{F}_{1}$-ATPase, protein kinase $\mathrm{C}$, calmodulin-dependent phoshodiesterase, and calcium activated $\mathrm{K}^{\prime}$-channel. ${ }^{15-18}$

In this paper, we have described an effect of dequalinium as a modifier of $A \beta$ aggregation by promoting and stabilizing the multimeric seed structures of $\mathrm{A} \beta$. By serving as a nucleation center, the $\mathrm{DQ}$-induced granular species gave rise to the $\mathrm{A} \beta$ aggregates which were different from the normal amyloid fibrils obtained in the absence of DQ in terms of their morphologies and cytotoxicities.

\section{Materials and Methods}

Materials. Human $\mathrm{A} \beta 40$ was purchased from SigmaAldrich Co. (St. Louis, USA) or Peptide Institute (Osaka, Japan). Dequalinium was obtained from Sigma. The carboncoated copper grid ( 300 mesh) and uranyl acetate were from Samchang Inc. and Electron Microscopy Sciences, respectively. Microcon-10 filter was purchased from Millipore Co. Human dopaminergic neuroblastoma cell line, SH-SY5Y, was a gift from Dr. Chung (Yonsei Univ., Korea). DMEM, trypsin-EDTA, and penicillin-streptomycin were provided by Gibco and fatal bovine senum was obtained from Hyclone. MTT, 3-[4,5-Dimethylthiazol-2-yl]-2,5-diphenyltetrazolium, for cell viability assay was purchased from Sigma. Materials used in this study were supplied from Sigma in the highest grade, unless otherwise mentioned. 
Preparation of $\mathbf{A} \bar{\rho}$ aggregates. $A \bar{\rho}$ was dissolved in $0.02 \% \mathrm{NH}_{3}$, and stored in aliquots at $-70{ }^{\circ} \mathrm{C}$. The dissolved peptide was diluted to $20 \mu \mathrm{M}$ in $20 \mathrm{mM}$ Mes, $\mathrm{pH} 6.5$, and incubated in the absence or presence of DQ without shaking at either $37^{\circ} \mathrm{C}$ or room temperature. Dequalinium stock was prepared in $50 \%$ ethanol at the highest concentration of 10 $\mathrm{mM}$ and diluted with $50 \%$ ethanol for subsequent use. The aggregative kinetics was monitored with thioflavin-T binding fluorescence at $485 \mathrm{~nm}$ with an excitation at $450 \mathrm{~nm}$. The thioflavin- $\mathrm{T}$ binding fluorescence of the $\mathrm{A} \vec{\beta}$ aggregates was obtained by combining the samples collected during the aggregative process and $2.5 \mu \mathrm{M}$ thioflavin- $\mathrm{T}$ in $50 \mathrm{mM}$ glycine at $\mathrm{pH} 8.5$.

Isolation of $\mathbf{D Q}$-induced granular species of $\mathbf{A} A$ Granular forms of $A \beta$ induced by dequalinium were isolated with a membrane filter of molecular weight cut-off limit at $10,000 \mathrm{Da}$. The peptide was dissolved in the $\mathrm{NH}_{3}$ and diluted with $20 \mathrm{mM}$ Mes, $\mathrm{pH} 6.5$, to a final concentration of $20 \mu \mathrm{M}$, which was incubated for 5 hours at $37^{\circ} \mathrm{C}$ in the presence of $100 \mu \mathrm{M} \mathrm{DQ}$. The filter was pre-washed with water and $20 \mathrm{mM}$ Mes buffer prior to the filtration. The peptide solutions were filtered through the pre-washed filter via a centrifugation at $12,000 \times \mathrm{g}$ for $20 \mathrm{~min}$.

Seed-dependent amyloid fibril formation of $\mathrm{A} \beta$ in the presence of the DQ-induced granular aggregates. The DQ-induced self-oligomerized granules were employed as the seed for the $\mathrm{A} \beta$ fibrillation. The granular species of $\mathrm{A} \beta$ obtained with $100 \mu \mathrm{M} D Q$ were added to $\mathrm{A} \beta$ monomer solution $(20 \mu \mathrm{M})$ at $3 \%$, and the mixtures were incubated at $37^{\circ} \mathrm{C}$. The aggregative kinetics was monitored with the thioflavin-T binding fluorescence.

Cell culture and cell viability test. Human dopaminergic SH-SY5Y cells were cultured in DMEM including $10 \%$ fetal bovine serum in the presence of penicillin-streptomycin at $37^{\circ} \mathrm{C}$ in $5 \% \mathrm{CO}_{2}$. The cells were subcultured whenever they reached $80 \%$ of confluency. Following trypsinization with $1 \mathrm{~mL}$ of trypsin-EDTA at $37^{\circ} \mathrm{C}$, the cells were plated in 96-well plate at $5 \times 10^{4}$ cells $/$ well and incubated for 48 hours. The cells were further incubated in the presence of the $\mathrm{A} / \beta$ amylojd fibrils at $37^{\circ} \mathrm{C}$ for overnight. Cell survival was evaluated with tetrazolium salt-extraction method. ${ }^{19}$ MTT, 3-[4,5-dimethyldiazol-2-yl]-2,5-diphenyltetrazolium bromide, was added to the culture medium at a final concentration of $1 \mathrm{mg} / \mathrm{mL}$ and incubated for 3 hours at $37^{\circ} \mathrm{C}$. The active mitochondria in living cells should cleave the tetrazolium ring into a visible dark-blue formazan product. To measure absorbance of the product at $570 \mathrm{~nm}$, MTT extraction buffer containing $20 \%$ SDS and $50 \% \mathrm{~N}, \mathrm{~N}$-dimethylformamide, $\mathrm{pH} 4.7$, was added to each sample $(100 \mu \mathrm{L}$ into $100 \mu \mathrm{L}$ medium in $96-w e l l$ plate including $20 \mu \mathrm{L}$ of $5 \mathrm{mg} /$ $\mathrm{mL}$ MTT) to dissolve the formazan grains. All the data related to the cytotoxicity were obtained from three separate experiments.

Miscellaneous. The $\mathrm{A} \beta$ aggregates were visualized with either a transmission electron microscope (TEM; H7100, Hitachi) or atomic force microscope (AFM; XE-150, PSIA). An aliquot $(5 u \mathrm{~L})$ containing the aggregates was adsorbed onto a carbon-coated copper grid ( 300 mesh) and air-dried for $1 \mathrm{~min}$. Following a negative staining with $2 \%$ uranyl acetate for $1 \mathrm{~min}$, the aggregates were examined under TEM. For AFM analysis, the aggregate-containing aliquot was placed on a freshly cleaved mica, and left for 1 to $2 \mathrm{~min}$ for the aggregates to be adsorbed. After displacement of the droplet with water and the removal of excessive water, the aggregates were analyzed with AFM.

Secondary structures of the $A \beta$ aggregates were monitored with circular dichroism (CD) spectroscopy (Jasco J-715) in $20 \mathrm{mM}$ potassium phosphate $(\mathrm{pH} 7.5)$ at $20^{\circ} \mathrm{C}$. The spectra were obtained within a $0.1-\mathrm{mm}$ pathlength quartz cell between $195 \mathrm{~nm}$ and $250 \mathrm{~nm}$ with a step resolution of 1.0 $\mathrm{nm}$, band width of $1.0 \mathrm{~nm}$, and scan speed of $20 \mathrm{~nm} / \mathrm{min}$. All of the spectra were obtained from an average of five scans.

\section{Results and Discussion}

Dequalinium effect on the amyloid fibril formation of $\mathrm{A} \beta$ has been examined. When incubated alone, $\mathrm{A} \beta$ showed a time-dependent increase in the thioflavin- $T$ binding fluorescence with a lag period of around 20 hours at $37^{\circ} \mathrm{C}$ (Fig. I). As DQ concentration increased, however, the final thioflavin-T binding fluorescence intensities became significantly suppressed in a dose-dependent manner. The decrease in the final fluorescence would indicate that the final $\mathrm{A} \beta$ aggregates have been produced to less amount. Altematively, the amyloid fibrils may have different distinctive structures from the cross $\beta$-sheet conformation which has been

A

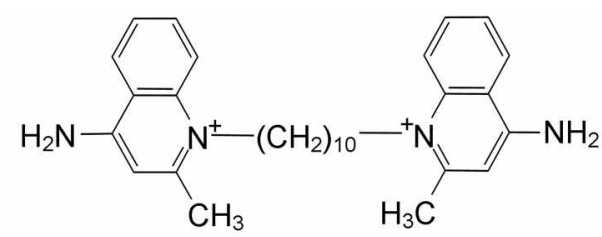

B

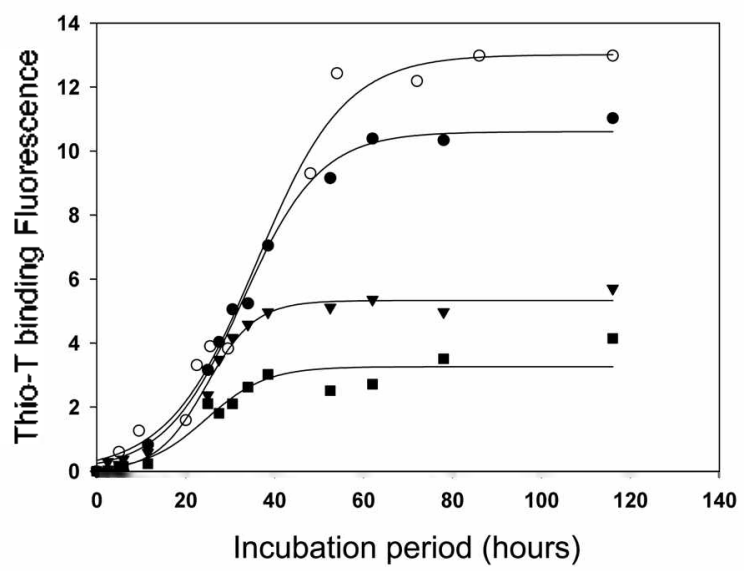

Figure 1. $N /$ aggregation kinctics in the presence and absence of DQ. ( $\Lambda$ ) Structure of dequalinium; (B) $\Lambda \beta$ prepared in $0.02 \% \mathrm{NH}_{3}$ was quiescently incubated at $20 \mathrm{pM}$ in $20 \mathrm{mM}$ Mes, $\mathrm{pH} 6.5$, at 37 ${ }^{\circ} \mathrm{C}$ in the absence $(\mathrm{O})$ and presence of various concentrations of $\mathrm{DQ}$ at $20 \mu \mathrm{M}(\bullet), 100 \mu \mathrm{M}(\boldsymbol{\nabla})$, and $200 \mu \mathrm{M}(\mathbf{\square})$. The aggregation kinctics was monitored with the thioflavin-T binding fluorescence at $485 \mathrm{~nm}$ with an excitation at $450 \mathrm{~nm}$. 
A

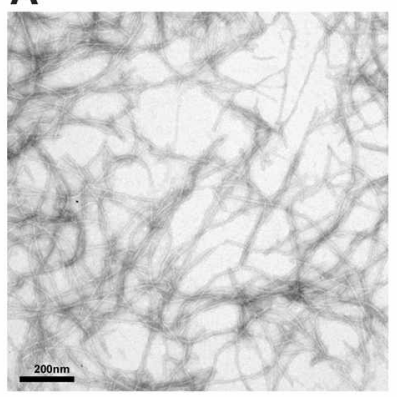

B
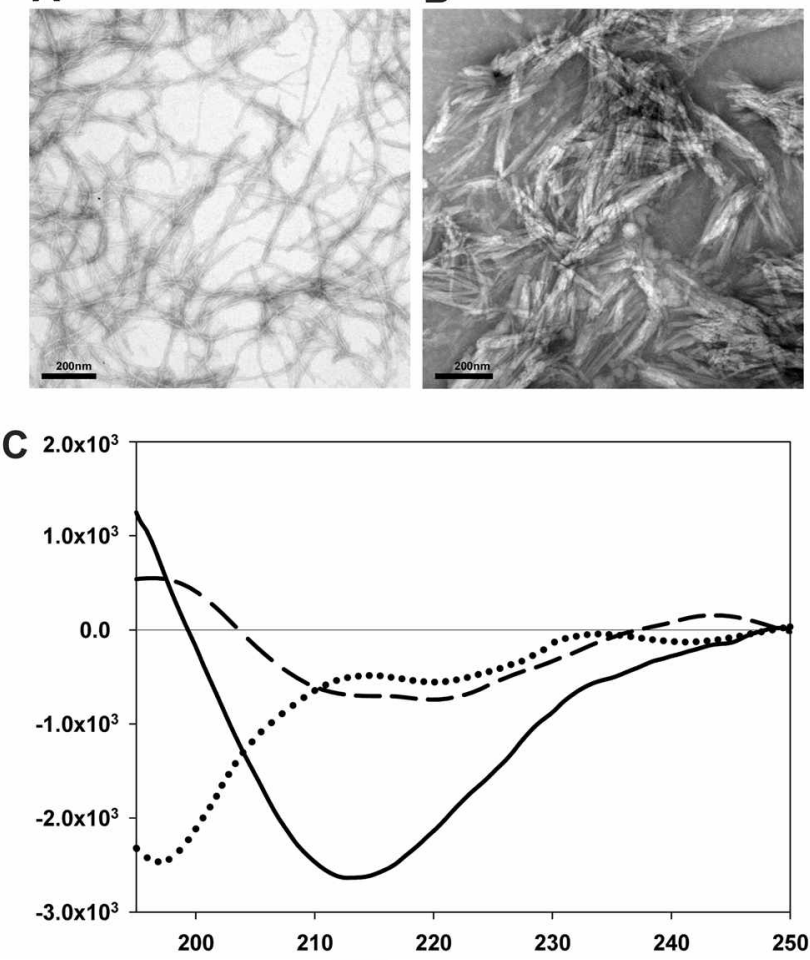

Figure 2. Morphologies of A/l aggregates prepared in the presence and absence of DQ and their secondary structure analyses. The final $A \beta$ aggregates obtained in the absence (A) and presence (B) of DQ were examined with a transinission clectron unicroscope following a negative staining with $2 \%$ uranyl acetate on a carboncoated copper grid (300 mesh). Secondary structures of the aggregates were monitored with circular dichroism spectroscopy in $20 \mathrm{mM}$ potassium phosphate $(\mathrm{pH} 7.5)$ at $20^{\circ} \mathrm{C}(\mathrm{C})$. Dotted, solid, and broken lines represent the spectra of the $\Lambda \beta$ mononer, the $\mathrm{A} \beta$ amyloid fibrils obtained without $\mathrm{DQ}$, and the $\mathrm{A} \beta$ aggregates prepared with DQ, respectively. suggested to accommodate thioflavin-T to exert the binding fluorescence.

To confirm the final states, transmission electron microscope and circular dichroism (CD) spectroscopy were employed to analyze the final fibrillar structures and the underlying secondary structures of the $A \beta$ aggregates, respectively. The $A \beta$ aggregates obtained in the absence of DQ showed a typical morphology of amyloid fibrils which appears thin single-stranded fibrils with clean surface under transmission electron microscope (Fig. 2A). In the presence of $\mathrm{DQ}$, however, the $\mathrm{A} \beta$ aggregates appeared to be choppedwood like structure with thick bundles of the fibrils (Fig. $2 \mathrm{~B}$ ). Secondary structures of the protein aggregates were also analyzed with $\mathrm{CD}$ spectroscopy. The $\mathrm{A} \beta$ monomer exhibited mostly the random structure with a minimum ellipticity at $197 \mathrm{~nm}$ (Fig. $2 \mathrm{C}$ ). As the $\mathrm{A} \beta$ fibrillation proceeded in the absence of $\mathrm{DQ}$, the obvious $\beta$-sheet conformation was induced from the previous random structure as the minimum ellipticity has shifted to $219 \mathrm{~nm}$ (Fig. 2C), which could be readily recognized by thioflavin-T binding fluorescence (Fig. 1). In the presence of DQ, however, the $\beta$ sheet content decreased as the minimum ellipticity at 219 nm monitored with the $\mathrm{A} \beta$ aggregates increased (Fig. 2C). It was, therefore, the final morphology with bundles of the fibrils and the altered underlying secondary structure of the $\mathrm{DQ}$-induced $\mathrm{A} \beta$ aggregates that should be responsible for the decreased thioflavin-T binding fluorescence as observed in Figure 1.

In order to examine molecular causes responsible for the dramatic alteration in the final structures of the $A \beta$ aggregates obtained in the presence or absence of $\mathrm{DQ}$, the morphological transition of $A \beta$ aggregates has been followed with AFM (Fig. 3). In the absence of DQ, the singlestranded $A \beta$ fibrils (Fig. $3 \mathrm{~b}$ and $3 \mathrm{c}$ ) have been gradually
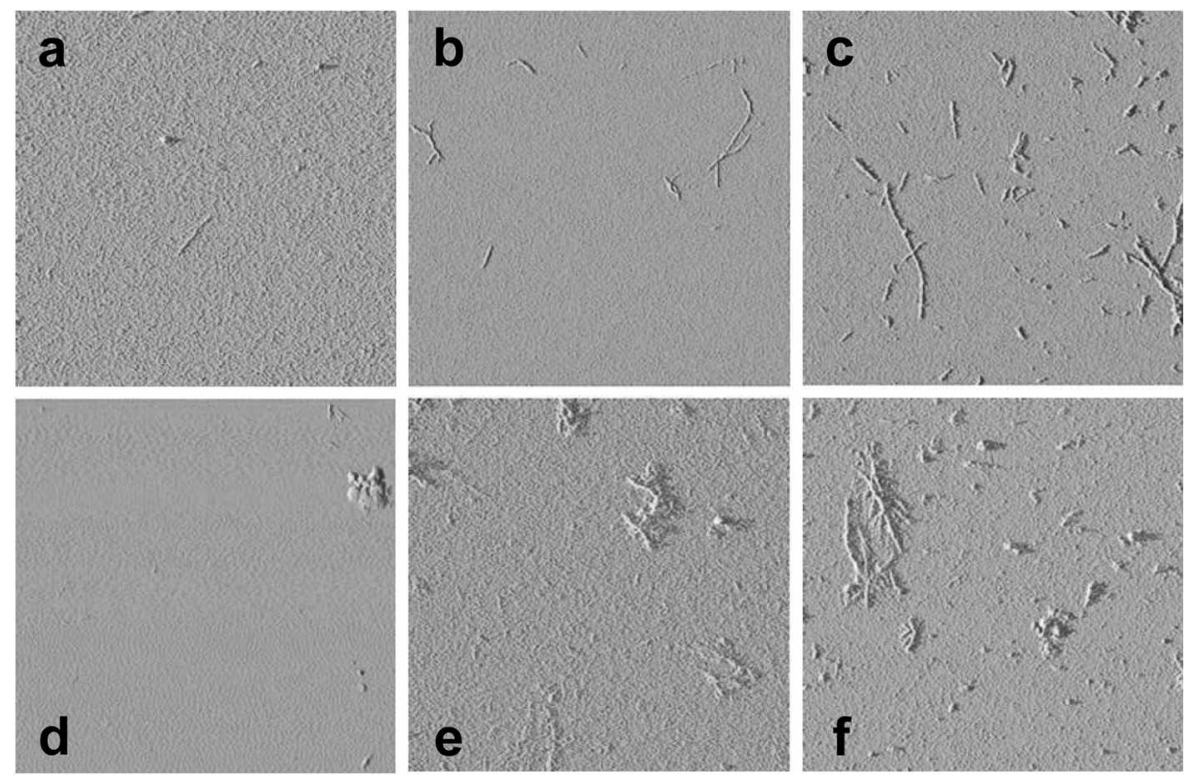

Figure 3. Structural transitions of $\mathrm{A} \beta$ aggregates obtained in the presence and absence of DQ. The $\mathrm{A} \beta$ aggregates collected at various time points during the aggregation kinetics in the absence $(a, b, c)$ or presence $(d, e, f)$ of $100 \mu \mathrm{M} D Q$ were examined with $\Lambda F M$. $\Lambda$ fter the aggregates were adsorbed on freshly cleaved mica with extra materials washed away, the aggregates were exaunined with $\Lambda F M$ on tapping mode. 
developed from the isolated round-shape granules as observed in Figure $3 \mathrm{a}$. In the presence of $\mathrm{DQ}$, however, the $\mathrm{A} \beta$ aggregates with multiple fibrils bundled together (Fig. $3 \mathrm{e}$ and $3 \mathrm{f}$ ) appeared to be induced from the initial peptide aggregates in which the round-shape granules were associated to form the multimeric structure (Fig. $3 \mathrm{~d}$ ). In other words, the bundled structure of $A \beta$ aggregates obtained with DQ-treatment has not been produced by lateral association of the preformed single-stranded fibrils, but instead they were resulted from the initial multimeric seed structures to which free $\mathrm{A} \beta$ has been accreted to multiple sites in parallel (Fig. 3e). Therefore, the initial seed structures of $A \beta$ aggregates should determine their final morphologies by providing appropriate nucleation centers. In other words, the seed effect could influence not only the aggregation kinetics by shortening the lag phase, but also the final morphology of the protein/peptide aggregates.

The seeding effect of the $\mathrm{DQ}$-induced initial $\mathrm{A} \beta$ aggregates has been investigated to assess whether they affect the final $\mathrm{A} \beta$ fibrillar structures as well as the aggregation kinetics. The seeds were prepared by incubating monomeric $\mathrm{A} \beta$ with $100 \mu \mathrm{M} \mathrm{DQ}$ for 5 hours at $37^{\circ} \mathrm{C}$ (Fig. $4 \mathrm{~A}$ inset).

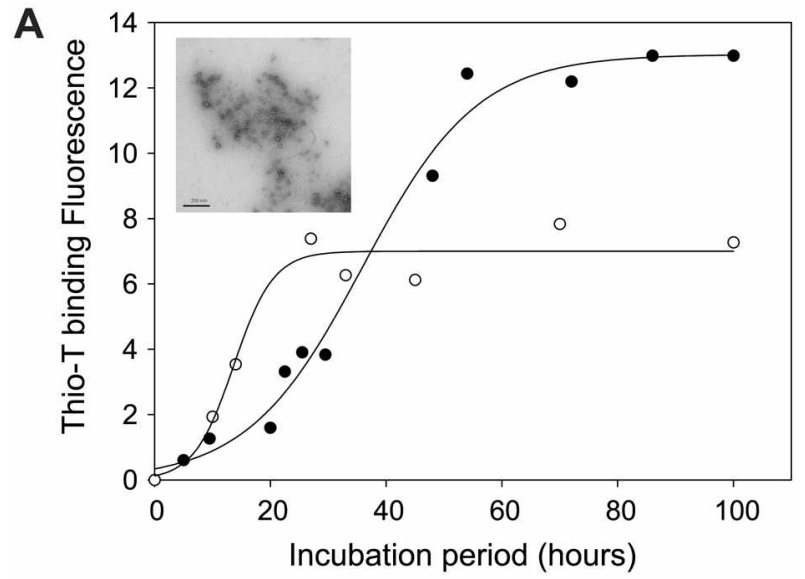

B

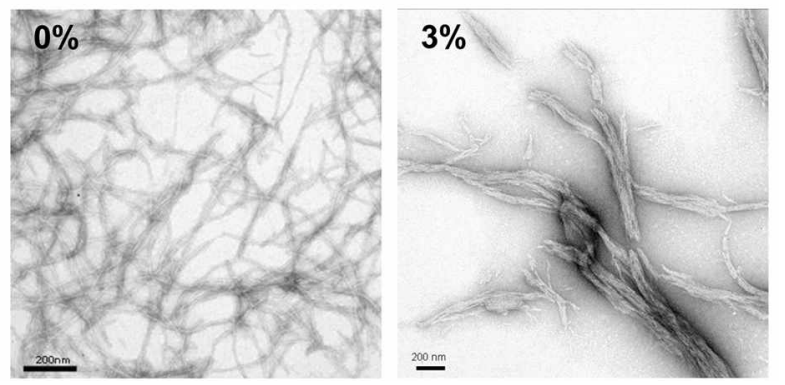

Figure 4. Sceding effect of the DQ-induced granular specics for $\mathrm{A} \beta$ aggregate formation. The DQ-induced gramlar species of $\mathrm{A} \beta$ were obtained by incubating the monomeric peptide $(20 \mu \mathrm{M})$ with $100 \mu \mathrm{M} \mathrm{DQ}$ in $20 \mathrm{mM} \mathrm{Mes}, \mathrm{pH} 6.5$, for 5 hours. The $\Lambda \beta$ aggregation was carried out in the presence ( $\circlearrowright$ ) or absence $(\bullet)$ of the granules to the amount of $3 \%$. The thioflavin-T binding fluorescence was monitored with the aliquots obtained during the aggregations at $485 \mathrm{~nm}$ with an excitation at $450 \mathrm{nun}(\Lambda)$. The final $\Lambda \beta$ aggregates collected without (B) and with (C) the seeds were revealed with TEM.
The seed was added into the A $\beta$ monomer solution at $20 \mu \mathrm{M}$ to the amount of $3 \%$, and incubated at $37^{\circ} \mathrm{C}$ for more than 100 hours. Compared with a control of the $\mathrm{A} \beta$ aggregation obtained in the absence of the seed, the lag period was significantly reduced, indicating that the $\mathrm{DQ}$-induced $\mathrm{A} \beta$ aggregates have exerted the seeding effect for the development of $A \beta$ aggregation. As expected from the DQ-induced $\mathrm{A} \beta$ aggregation, the final thioflavin-T binding fluorescence was also significantly suppressed as the seeds were included in the aggregation mixture (Fig. 4A). The final morphology of the seed-induced $A \beta$ aggregates was distinctive from the nomnal amyloid fibrils of $A \beta$ obtained in the absence of the seeds (Fig. $4 \mathrm{~B}$ and $C$ ). Those seeded-aggregates appeared more like the $\mathrm{DQ}$-induced $\mathrm{A} \beta$ aggregates with the bundled fibrillar structures (Fig. $4 \mathrm{C}$ ). The data clearly shows that the altered morphology of the $\mathrm{DQ}$-induced $\mathrm{A} \beta$ aggregates could be due to the initial $A \beta$ aggregates which act as the seeds during the $A \beta$ aggregation. In other words, the final morphology of $\mathrm{A} \beta$ amyloid fibrils could be manipulated by modifying the seed structures.

The cytotoxicities of the $\mathrm{A} \beta$ aggregates prepared in the absence and presence of DQ were monitored with SH-SY5Y cells employing MTT assay. While the normal $\mathrm{A} \beta$ amyloid fibrils exerted the cytotoxicity by $28 \%$, the seeded-A $\beta$ aggregates showed the toxicity by only $12 \%$ (Fig. 5 ). The reduced cytotoxicity seemed to be due to the altered morphology of the seeded-aggregates since polymorphism of the amyloid fibrils has been suggested to be responsible for the varied cellular toxicities. ${ }^{20-22}$ This data indicates that the cytotoxicity of amyloids could be controlled by manipulating the seed structures which would lead to multiplicity

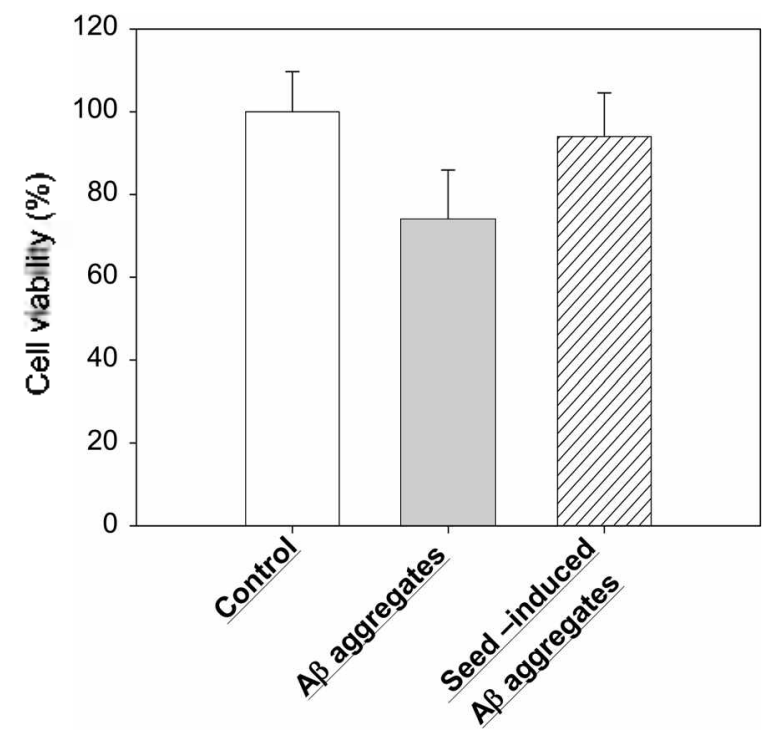

Figure 5. Cytotoxicities of the $A \beta$ aggregates generated with and without the DQ-induced seeds. The cell death of human dopaninergic SH-SY5Y cells was monitored with MTT assay in the presence of the $A / \beta$ aggregates obtained without (gray bar) and with (oblique bar) the DQ-induced seeds at 3\% (Fig. 4B and C). The control cell survival has been shown in white bar. The cell viability for cach condition was estimated with three separate expcriments. 
in the amyloid fibrils in shapes.

In fact, a number of different therapeutic strategies toward $\mathrm{AD}$ have been proposed in relation to $\mathrm{A} \beta{ }^{23,24}$ which include a suppressed $\mathrm{A} \beta$ production, elimination of the existing amyloids from the affected brain, and blocking of the initial conformational change of $\mathrm{A} \beta$ to its pathological $\beta$-sheet structure and subsequent transition to oligomers, protofibrils, or fibrils. Several small molecules have been shown to be capable of inhibiting $A \beta$ aggregation, including enoxaparin, nicotine, and curcumin. ${ }^{2022}$ In this respect, DQ examined in this study is unique since the compound has changed eventual fibrillar structures exerting varied cytotoxicities by altering the seed structures via facilitated association of the initial granular aggregates. In other words, DQ would exhibit its regulatory effect on the cytotoxicity of $\mathrm{A} \beta$ aggregates by changing the final morphology while other compounds have been suggested to play therapeutic roles simply by inhibiting the aggregation process. Therefore, DQ has been proposed to be a useful chemical probe to control the cytotoxicity of the amyloid fibrils.

In conclusion, this investigation provides information that the seeds have acted as templates for the $\mathrm{A} \beta$ aggragation which could determine the final morphology of the aggregates and influence the aggregation kinetics by shortening of the lag period as well. In addition, the cytotoxicity of the $\mathrm{A} \beta$ aggregates has been also varied depending on their morphologies. It is, therefore, the seed structures that could be central to develop therapeutic strategy by inducing the amyloid fibrils in different shapes with varied cytotoxicities.

Acknowledgements. This study was supported in part by Grant from the Seoul R\&BD Program (10538) and another grant from Technology Development Program of the Ministry of Agriculture and Forestry.

\section{References}

1. Coyle, J. T.; Price, D. L.; DeLong, M. R. Science 1983, 219(4589),
1184.

2. Bourin, M.; Ripoll, N.; Dailly, E. Curr. Med. Res. Opin. 2003, 19, 169.

3. Tanzi, R.; Bertram, L. Cell 2005, 120(4), 545.

4. Jarrett, J. T.; Berger, E. P.; Lansbury, P. T. Jt. Biochemistry 1993, $32(18), 4693$.

5. Selkoe, D. J. Nature 1999, 399, A23.

6. Younkim, S. G. Ann. Netrol. 1995, 37, 287.

7. Hardy, J. A.; Selkoe, D. J. Science 2002, 297(5580), 353.

8. Yang, D. S.; Yip, C. M.; Huang, T. H.; Chakrabarty, A.; Fraser, P. E. J. Biol. Chem, 1999, 274(46), 32970.

9. Williams, A. D.; Sega, M.; Chen, M.; Kheterpal, I.; Geva, M.; Berthelier, V.; Kaleta, D. T.; Cook, K. D.; Wetzel, R. Proc. Natl. Acod. Sci. USA 2005, 102(20), 7115.

10. Necula, M.; Kayed, R.; Milton, S.; Glabe, C. G. J. Biol. Chent. 2007, 282(14), 10311 .

11. Diamant, S.; Podoly, E.; Friedler, A.; Ligumsky, H.; Livnah, O.; Soreq, H. Proc. Natl. Acad. Sci. USA 2006, 103(23), 8628.

12. Marambaud, P.; Zhao, H.; Davies, P. J. Biol. Chem. 2005, 280(45), 37377.

13. Lee, C.-H.; Kim, H. J.; Lee, J.-H.; Cho, H.-J.; Kim, J.; Chung, K. C.; Jung, S.; Paik, S. R. J. Biol. Chem. 2006, 281/6), 3463.

14. Weiss, M. J.; Wong, J. R.; Ha, C. S.; Bleday, R,; Salem, R. R; Steele, G. D.; Chen, L. B. Proc. Natl. Acad. Sci. USA 1987, 84, 5444.

15. Zhuo, S.; Allison, W. S. Biochem. Biophys. Res. Commun. 1988 , I52, 968 .

16. Zhuo, S.; Paik, S. R.; Register, J. A.; Allison, W. S. Biochenistry $1993,32,2219$

17. Rotenberg S. A.; Smiley, S.; Ueffing, M.; Krauss, R. S.; Chen, L. B.; Weinstein, I. B. Cancer Res. 1990, 50, 677.

18. Sullivan, R. M.; Stone, M.; Marshall, J. F.; Uberall, F.; Rotenberg, S. A. Mol. Pharmacol. $2000,58,729$.

19. Petkova, A. T.; Leapman, R. D.; Guo, Z.; Yau, W. M.; Mattson, M. P.; Tycko, R. Science 2005, 307(5707), 262.

20. Bergamaschini, L.; Rossi, E.; Storini, C.; Pizzimenti, S.; Distaso, M.; Perego, C.; De Luigi, A.; Vergani, C.; Grazia De \$imoni, M. J. Netrosci. 2004, 24, 4181 .

21. Ashur-Fabian, O.; Segal-Ruder, Y.; Skutelsky, E.; Brenneman, D. E.; Steingart, R.; Giladi, E.; Gozes, I. Peptides 2003, 24 , 1413.

22. Ono, S.; Mohri, K.; Ono, K. J. Biol. Chem, 2004, $279(14), 14207$.

23. Koh, C. J.; Lee, M. Bull. Korean Chem. Soc. 2006, 27(4), 477.

24. Kang, D.-I.; Baek, D.; Shin, S. Y.; Kim, Y. Bttl. Korean Chen. Soc. 2005, 26(8), 1225 . 